\title{
Book Review: An Introduction to Music Therapy Research
}

\author{
Lori Fogus Gooding ${ }^{1 *}$ \\ 1 Florida State University, United States \\ *lgooding@fsu.edu \\ Received: 12 October 2017 ; Accepted: 27 November 2017 ; Published: 1 March 2018 \\ Editor: Melody Schwantes
}

\begin{abstract}
An Introduction to Music Therapy Research, edited by Barbara Wheeler and Kathleen Murphy, is a 20-chapter that provides overview of how research has been conceived and implemented in music therapy. The text is geared to those beginning their studies in music therapy and as such address all stages of research, beginning with foundational aspects like selection of a topic before moving to a more detailed presentation of specific research components like data analysis and interpretation of results. Objectivist (qualitative) and interpretivist (quantitative research) theoretical perspectives, methodologies, and methods are included in the book, as well as information on mixed methods research and important historical, ethical, and multicultural considerations.
\end{abstract}

Keywords: music therapy research, objectivist, interpretivist

\section{Music therapy research (3rd edition)}

Edited by Barbara L. Wheeler \& Kathleen M. Murphy. 253 pages. Dallas, Barcelona Publishers, 2016. \$42.00 (paperback). ISBN: 9781945411120

Connecting music therapists with research is key to advancing practice, and those connections start with accessing and understanding the fundamental concepts of research. Barbara Wheeler has been engaged in educating others about music therapy research for over 20 years, and her latest book, An Introduction to Music Therapy Research, continues along that path. Wheeler, along with associate editor Kathleen Murphy, have oriented the text toward those beginning their studies in music therapy and as such address all stages of research, beginning with foundational aspects like selection of a topic before moving to a more detailed presentation of specific research components like data analysis and interpretation of results. The book contains 20 chapters contributed by over 15 authors, 17 of which are excerpted from Wheeler and Murphy's larger work, Music Therapy Research (2016). Throughout the text Wheeler and Murphy provide an "overview of how research has been conceived and implemented in music therapy" (Bruscia, p. xiii), taking careful consideration to include objectivist (quantitative) and interpretivist (qualitative) theoretical perspectives, methodologies, and methods, as well as information on mixed methods research and important historical, ethical, and multicultural considerations. 
An Introduction to Music Therapy Research is only slightly smaller than Wheeler's original 1995 text Music Therapy Research: Quantitative and Qualitative Perspectives, which speaks volumes about the increasing knowledge base of music therapy research. While the aforementioned Music Therapy Research (2016), a 758-page resource, could be overwhelming to those new to music therapy research, this condensed text takes a more measured approach, beginning with elemental aspects of the research process (e.g., Darrow's chapter on developing a topic and Murphy's chapter on ethical thinking) before exploring research paradigms in more detail (e.g., Cohen's and Wheeler's respective chapters on the principles of objectivist and interpretivist research and McKinney's and Abrams' respective chapters on evaluating objectivist and interpretivist research). As a music therapy educator, I found the information both practical and useful, and I can easily envision how this text could be integrated across the music therapy curriculum. For example, Abbott's chapter (4) on reviewing the literature provides practical guidance for students who are just beginning to explore the research literature, while Meadows' and Gardstrom's chapters (19 and 20, respectively) walk readers through the process of reading-and interpreting-the research. These chapters could be paired to guide those newly engaged with music therapy research as they develop the ability to investigate and understand the existing literature. Those with more research experience could also find applicability in this text as it provides submission guidance (chapters 19 and 20) and challenges the reader to consider important ethical (chapter 5) and multicultural considerations (chapter 6). In this way Wheeler and Murphy's text has great utility, with relevance for those at different points in their research journey. However, I would recommend the more comprehensive Music Therapy Research (2016) for experienced researchers, as the greater breadth and depth may be more in line with their needs.

The bulk of the text alternates between objectivist and interpretivist chapters, presenting foundational concepts from both paradigms. On one hand, this alternating approach helps to equate the differing orientations, highlighting the engagement by music therapy researchers in a broad spectrum of research and challenging readers to allow the research question to guide their use of a particular research paradigm. On the other hand, this approach has the potential to present challenges for novice researchers who may not have developed a comprehensive understanding of the foundational concepts in one or more paradigms. While I appreciate the attempt to counterbalance the presentation of differing research paradigms, I felt like clarity was lost as I maneuvered between orientations and chapters, particularly when interacting with chapters in which the concepts were less familiar and/or when detailed descriptions were not provided. (There were several instances where concepts were introduced but detailed descriptions were not provided until subsequent portions of the text. For example, Type I and Type II errors are discussed in Chapter 11 ["Potential Problems in Objectivist Research"], but the concepts are not fully explained until Chapter 12 ["Introduction to Statistical Concepts"].) This does not negate the usefulness of the text, but instead suggests that this condensed volume is best suited to serve as a supplement to classroom instruction or paired with guidance from experienced researchers.

I appreciate Wheeler and Murphy's endeavor to make music therapy research more accessible through their circumspect construction of the book, the reasonable cost of the text, and the multi-format availability. As mentioned previously, this text has applicability for coursework with and without research as the primary focus, and the potential for cross-curriculum integration is the most salient aspect of Wheeler and Murphy's work. An Introduction to Music Therapy Research is a welcome addition to the music therapy literature.

\section{References}

Wheeler, B. L. (1995). Music therapy research: Quantitative and qualitative perspectives. TX: Barcelona Publishers. 
Wheeler, B. L., \& Murphy, K. M. (Eds.). (2016). Music therapy research (3rd ed.). Dallas, TX: Barcelona Publishers. 